\title{
Maximizing Downlink Bandwidth Allocation Method Based on SVC in Mobile WiMAX Networks for Generic Broadband Services
}

\author{
I-S. Hwang, ${ }^{1}$ Bor-Jiunn Hwang, ${ }^{2}$ and Ruey-Rong Su${ }^{1,3}$ \\ ${ }^{1}$ Department of Computer Science and Engineering, Yuan Ze University, Chung Li, 32003, Taiwan \\ ${ }^{2}$ Department of Computer and Communication Engineering, Ming Chuan University, TaoYuan 33348, Taiwan \\ ${ }^{3}$ Department of Information Management, Kang-Ning Junior College of Medical Care and Management, Taipei 114, Taiwan \\ Correspondence should be addressed to I-S. Hwang, ishwang@saturn.yzu.edu.tw
}

Received 31 August 2010; Accepted 4 October 2010

Academic Editors: G. Kim and Y.-J. Suh

Copyright (๑) 2011 I-S. Hwang et al. This is an open access article distributed under the Creative Commons Attribution License, which permits unrestricted use, distribution, and reproduction in any medium, provided the original work is properly cited.

This paper proposes an adaptive downlink bandwidth allocation method (DBAM) for six traffic types-UGS, RT-VR, ERT-VR, NRT-VR, BE, and multicast - to maximize the throughput of broadband WiMAX networks for generic broadband services. Based on traffic throughput and the amount of traffic in different scalable video coding (SVC) layers, two adaptive resource adjustment schemes with SVC technology in the DBAM are proposed and compared. Moreover, a hierarchical priority queuing for different traffic profiles with weighted round robin (HPWRR) scheduling algorithm is proposed to achieve higher resource utilization and to meet the required quality of service (QoS) for each traffic type. Simulation results show that the proposed DBAM with HPWRR can achieve efficient throughput and bandwidth utilization and improve the delay time for RT-VR and multicast traffic.

\section{Introduction}

Existing wireless technologies are struggling to satisfy the enormous growth in demand for broadband wireless access (BWA), anytime and anywhere. Mobile worldwide interoperability for microwave access (WiMAX) systems, which is defined by the IEEE 802.16e standard [1] and called a broadband wireless metropolitan area network (WMAN), is an emerging technology. In mobile WiMAX, the IEEE 802.16e standard supports multicast service with different requirements of quality of service (QoS) and multirate transmission techniques with link adaptive capability. Since the QoS of the mobile station will be affected by the received signal-to-noise ratio (SNR), the mobile WiMAX BS determines the appropriate modulation technique based on the received and required SNRs. Basically, the IEEE 802.16e defines several burst profiles-combinations of modulation and coding schemes - in each PHY configuration to improve the total system throughput.

Multicast is known as a bandwidth-conserving technology that reduces traffic by delivering the same data stream to multiple recipients simultaneously. The subscriber stations (SSs) interested in receiving the data stream are included in the related multicast group and referred to as multicast group members [3]. The IEEE 802.16e standard defines the multicast broadcast service standard for current broadcast services and supports the QoS requirements. In [4], Alanen proposed an optimal multicast polling technique feature applied to the base station that achieves better, and even guaranteed, quality of service. However, multicast polling is only allowed for NRT-VR, ERT-VR, and BE connections; it does not support RT-VR traffic and increases the overhead of MAC layers when operators adjust the network parameters. In [5], accurate analytical models of the mechanism with various leader selection schemes, based on the concept of multiple ACK leaders, are proposed for multicast services in WiMAX networks; but they provide insufficient reliability. Challenges are posed for IPTV over WiMAX due to multicast under a diversity of fading conditions [6]. A cross-layer design framework, based on the two-level superposition coded multicasting (SCM) scheme, is introduced, and the challenge is to extend IPTV services in wireless and mobile network environments. In [7], to maximize total utility, the paper proposed a utility-based resource allocation scheme for layer-encoded IPTV multicast streaming service over WiMAX networks, which adjusts the number of received 
layers for each user dynamically, according to the channel conditions and the available network bandwidth. However, when a multicast video stream is transmitted, all SSs distributed over different locations (i.e., with different SNRs) receive the data stream using the lowest-order modulation or the coding rate based on the lowest $\mathrm{SNR}$ - even though some SSs have better SNR which will waste the system bandwidth and degrade the system throughput [8]. Additionally, an MN (mobile node) can move and get a higher SNR; thus, it can change to a higher-order modulation or coding rate. However, when an MN changes from using a higher-order modulation or coding rate to lower one, this reduces the transmission quality. In these two cases, if the system cannot allocate resources or adjust the transmission rate adaptively, it will waste resources (i.e., the former case) or the call will be blocked, resulting in lower quality (i.e., the latter case).

To support higher service quality given the limited bandwidth, it is desirable to adapt the video signal bit rate. To achieve flexible bit stream adaptation for diversified transmission conditions, the Joint Video Team (JVT), formed by the ISO/IEC MPEG and ITU-T, developed the H.264/MPEG4 SVC (scalable video coding) technology, which provides different resolutions in spatial scalability, various frame rates in temporal scalability, and the visual quality is scalable according to the SNR $[8,9]$. One SVC-based video stream consists of a base layer (BL) and several enhancement layers (ELs). In the link adaptive mobile WiMAX system, because multicast services adopt SVC technology, the system performance increases to maximize the system resources, in terms of channel utilization [10-12]. In [10], the authors give an overview of SVC and its relation to mobile delivery methods and propose innovative uses, including (a) wireless multiuser video streaming using SVC, (b) distributed video streaming in mobile multihop networks, and (c) scalable on-demand services over broadcast channels. The SVC technology is applied to the SVC server to achieve graceful degradation of the video quality, rather than visible errors or interruptions in the wireless network [11]. In [12], the authors analyze the performance of a hierarchical video ondemand $(\mathrm{VoD})$ system using layered videos in a broadcast environment, which not only stores popular videos in the proxy server, but also broadcasts some of the video layers to the customers to minimize the blocking probability of the system. Both adaptive modulation coding (AMC) and SVC are employed to enhance the perceived video quality, and the intermediate control server (ICS) deals with the signals between the multimedia server and the BS to maximize the resource utilization [13]. However, this approach considers only the IPTV traffic and does not specify the scheduling for other traffic types. Moreover, the SVC technologies can be integrated with multicast services using a fluctuating bandwidth and higher utilization rate in the scheduling algorithms.

A user roaming under a cell, defined as a moving call, will change modulation to achieve higher system throughput or meet a required QoS. Thus, new calls, handoff calls, and moving calls should be taken into account for the radio resource management strategy to achieve optimum results. For moving or handoff calls, the resources would be inadequate if the user is moving from a lower modulation rate to a higher one; resources would be wasted if the user were moving from a higher modulation rate to a lower one. This paper adopts SVC technology to overcome problems of resource deficiencies or waste. Based on the received SNR of each SS, the BS adjusts the burst profile and applies SVC technology to improving the visual quality and system throughput. An efficient scheduling algorithm is important for optimizing the quality of services based on the different traffic types and locations to improve system throughput in mobile WiMAX networks $[14,15]$. In addition to the five traffic types, UGS, RT-VR, NRT-VR, ERT-VR, and BE, defined in the IEEE 802.16e standard, the multicast session is proposed as an independent traffic type applied with SVC technology.

In this paper, the BS determines the allocated bandwidth using downlink bandwidth allocation method (DBAM) when a WiMAX new call, moving call, or handoff call request arrives. Based on the properties of the transmitted traffic and the system status, two adaptive resource adjustments schemes in the DBAM are proposed and compared. The hierarchical priority queuing with weighted round robin (HPWRR) scheduling algorithm for different traffic profiles and multicast session traffic is proposed to achieve higher resource utilization and to meet each required QoS. The rest of the paper is organized as follows. Section 2 describes the system architecture and the proposed methods. The simulation model and performance evaluation are presented in Section 3. Section 4 concludes the paper and suggests future work.

\section{System Architecture and the Proposed Method}

Figure 1 shows the system architecture; mobile users access the network resource by the WiMAX BS; the users make new calls, handoff calls, and moving calls. Users request access to the network with different burst profiles.

The procedure for new, handoff, or moving calls is shown in Figure 2. When a call arrives, the BS categorizes the user by the burst profile and estimates the allocated bandwidth with the DBAM; then the call admission control decides whether the call is accepted or rejected, based on the current resources. The traffic is processed by the hierarchical priority queuing with weighted round robin (HPWRR) scheduling algorithm once the call is accepted by the call admission control mechanism or a moving call arrives.

2.1. Downlink Bandwidth Allocation Method (DBAM). This section describes the DBAM, shown in Algorithm 1, which allocates six traffic types: UGS, RT-VR, ERT-VR, NRT-VR, $\mathrm{BE}$, and multicast. Based on the priority of traffic type, the system allocates a bandwidth with a sustained rate for UGS and ERT-VR traffic first. RT-VR traffic, such as real time MPEG video traffic, is classified as unicast or multicast. For unicast RT-VR traffic, the bandwidth is assigned according to the worst-case delay time [16] — the BS allocates bandwidth for the unicast using a leaky bucket algorithm with the 


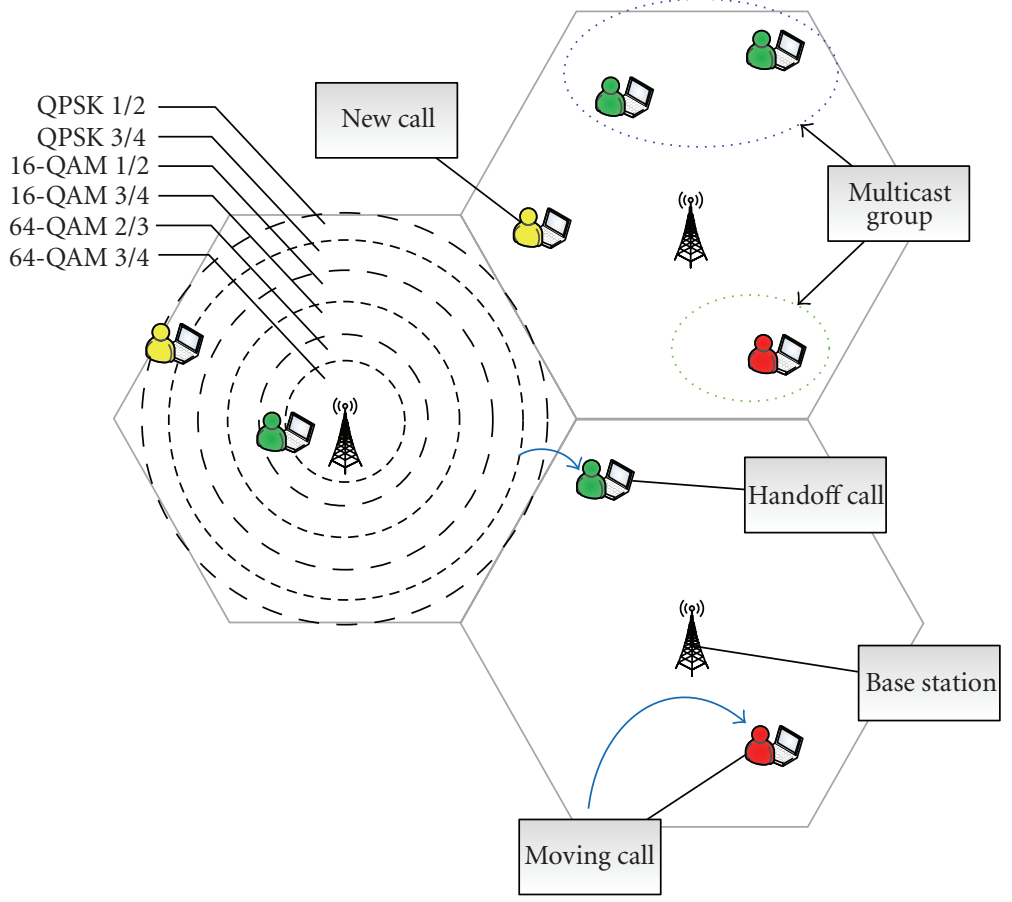

Figure 1: System Architecture.

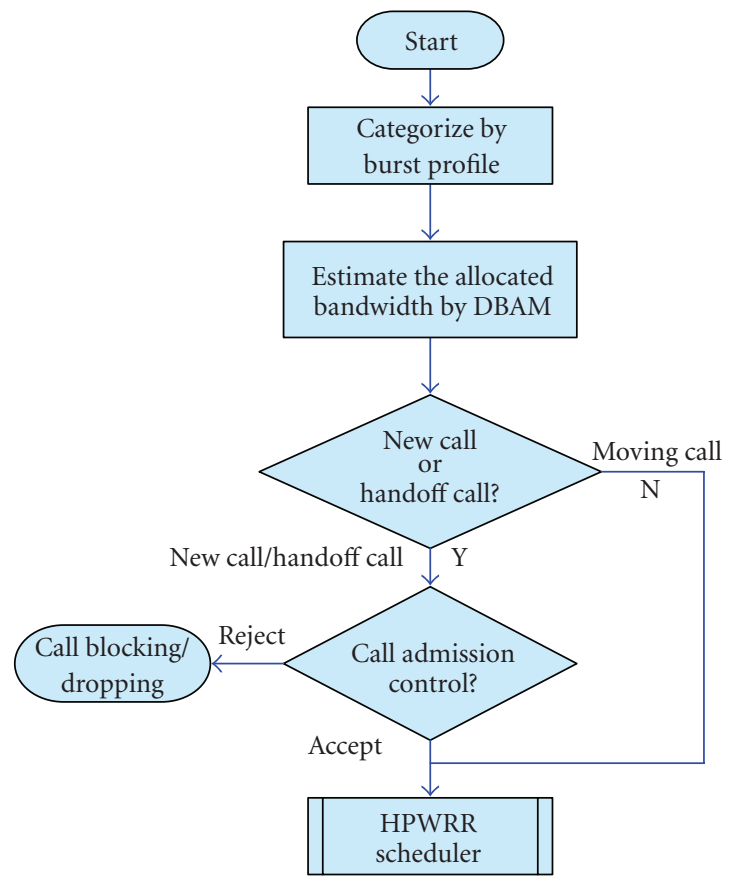

Figure 2: Procedure for new/handoff/moving calls.

downlink adaptive adjustment, as shown in Algorithm 2. A multicast session is transmitted with layered video streams to provide different video qualities by transmitting different numbers of layers flexibly, depending on the available bandwidth and user burst profiles. Additionally, for efficient transmission, users using QPSK can only decode the base layer, users using 16-QAM can decode the base layer and enhancement layer I, and users using 64-QAM can decode the base layer data and enhancement layers I and II. The users access the multicast data of the different multicast groups based on the multicast ID (MCID). The BS allocates bandwidth for multicast users based on their burst profiles. For the NRT-VR traffic, such as FTP applications, the BS allocates bandwidth based on the minimum required rate. For BE traffic, the BS allocates the bandwidth based on the excess bandwidth. For each traffic type, we proposed the DBAM algorithm to maximize the downlink allocation bandwidth using scalable video code technologies especially for the multicast traffic.

As shown in Algorithm 2, the downlink adaptive adjustment contains the downlink adaptive bandwidth Schemes 1 (DABS1) and 2 (DABS2). DABS1 and DABS2 adjust the resources based on traffic throughput and the amount of traffic in the different SVC layers, respectively.

2.1.1. Downlink Adaptive Bandwidth Scheme 1 (DABS1). To optimize the bandwidth and improve the system throughput, the bandwidth for the video stream is transmitted by the proportion ratio to data rate of the $Q_{64-\mathrm{QAM} / 4}$ which is the main idea of DABS1. From the point of view of throughput, when the same number of OFDM symbols is allocated, adopting 64-QAM 3/4 provides a throughput nine times higher than that with BPSK $1 / 2$. Therefore, the number of OFDM symbols needed to provide the determined throughput is based on the modulation technique. Based on the number of bytes attainable in one slot for the different MCSs [17], the proposed method adjusts the allocation 
Downlink Bandwidth Allocation Method (DBAM)

IF call traffic $=$ UGS or ERT-VR traffic

Calculate bandwidth according to sustained rate.

Allocate bandwidth.

IF call traffic $=$ RT-VR traffic

IF call traffic $=$ unicast

Calculate the bandwidth by leaky bucket algorithm and downlink adaptive adjustment.

Allocate bandwidth.

ELSE IF call traffic $=$ multicast

IF user burst profile $=64$-QAM

According the burst profile assigns MCID, and the SS can access base layer and two enhancement layers.

ELSE IF user burst profile $=16$-QAM

According the burst profile assigns MCID, and the SS can access base layer and one enhancement layer.

ELSE IF user burst profile $=$ QPSK

According the burst profile assigns MCID, and the SS can only access base layer.

IF call traffic $=$ NRT-VR traffic

The bandwidth allocated to NRT-VR based on minimum required rate

IF call traffic $=$ BE traffic

IF excess bandwidth $=$ True Allocate bandwidth.

Algorithm 1: Downlink bandwidth allocation method for different traffic profiles.

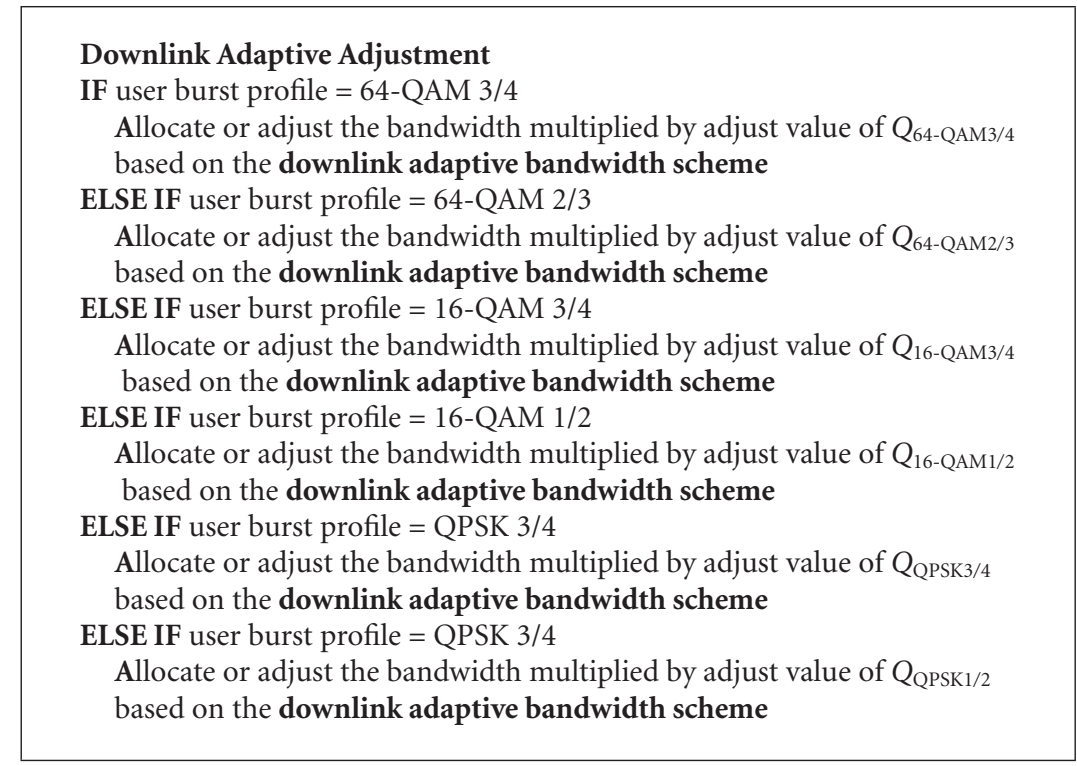

Algorithm 2: Downlink adaptive adjustment for different traffic profiles.

bandwidth by multiplying the adaptive parameter defined in Table 1 from different burst profiles.

2.1.2. Downlink Adaptive Bandwidth Scheme 2 (DABS2). For DABS2, the video transmitted by the ratio of the amount of traffic for adaptive layers to the amount of traffic for total layers, as shown in Table 2. Assume that the base layer is $x \%$ of the total SVC data, the enhancement layer I is $y \%$ of total SVC data, and the enhancement layer II is $z \%$ of total SVC data [2]. For example, transmitting
TABLE 1: Value of adaptive parameter in DABS1.

\begin{tabular}{lcc}
\hline Parameter & Downlink rate $(\mathrm{Mbps})$ & Value \\
\hline$Q_{\mathrm{QPSK} 1 / 2}$ & 6.34 & 0.22 \\
$Q_{\mathrm{QPSK} 3 / 4}$ & 9.50 & 0.33 \\
$Q_{16-\mathrm{QAM} 1 / 2}$ & 12.67 & 0.44 \\
$Q_{16-\mathrm{QAM} 3 / 4}$ & 19.01 & 0.66 \\
$Q_{64-\mathrm{QAM} 2 / 3}$ & 25.34 & 0.88 \\
$Q_{64-\mathrm{QAM} 3 / 4}$ & 28.51 & 1.00 \\
\hline
\end{tabular}




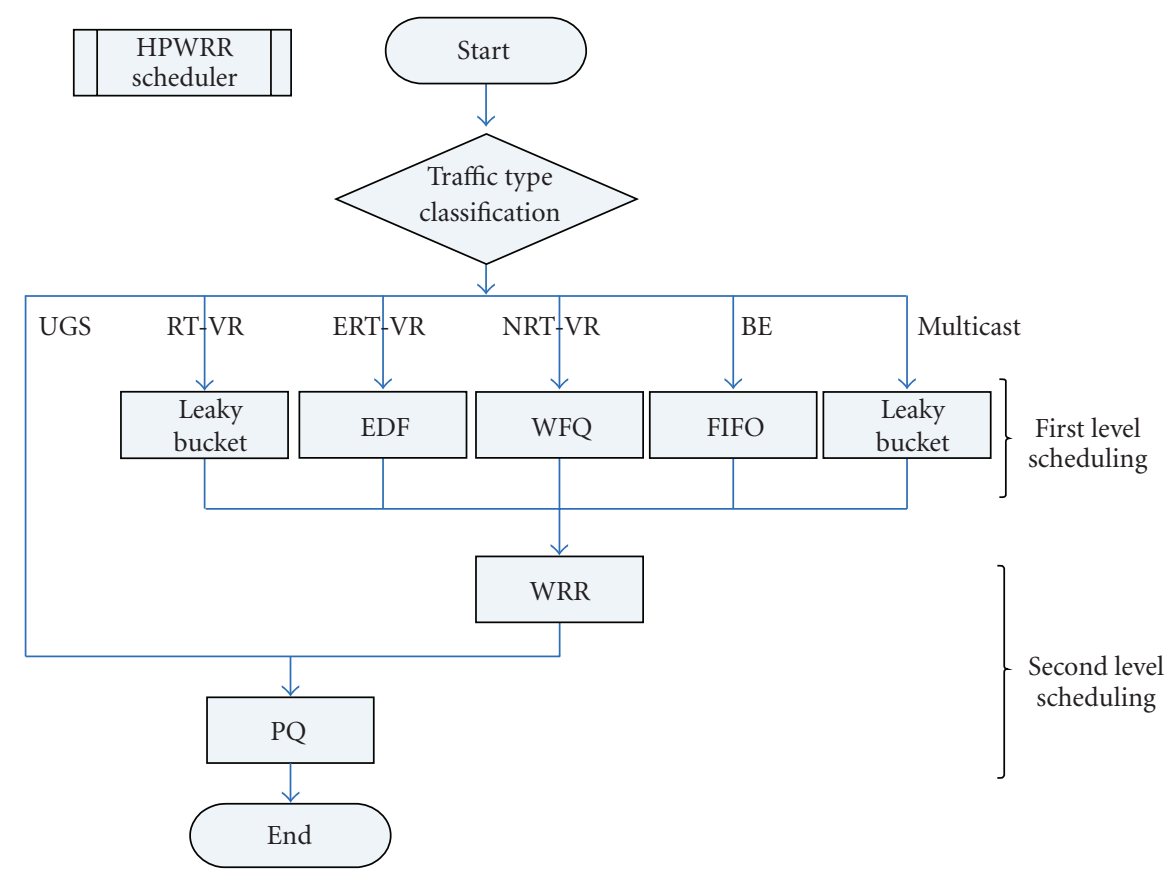

FIGURE 3: HPWRR scheduling architecture.

TABLE 2: Value of adaptive parameter in DABS2.

\begin{tabular}{lcc}
\hline Parameter & Ratio & Value \\
\hline$Q_{\mathrm{QPSK} 1 / 2}$ & $\frac{x}{x+y+z}$ & 0.655 \\
$Q_{\mathrm{QPSK} 3 / 4}$ & $\frac{x+y}{x+y+z}$ & \\
$Q_{16-\mathrm{QAM} 1 / 2}$ & $\frac{x+y+z}{x+y+z}$ & \\
$Q_{16-\mathrm{QAM} 3 / 4}$ & 1.863 \\
$Q_{64-\mathrm{QAM} 2 / 3}$ & $Q_{64-\mathrm{QAM} 3 / 4}$ & \\
\hline
\end{tabular}

the common intermediate format (CIF) with $15 \mathrm{fps}$ (frame per second) requires $256 \mathrm{kbps}$ [18]. If the length of the group of pictures (GOP) is 15 and the GOP structure is IBBPBBPBBPBBPBB, then the ratio of $\mathrm{I}, \mathrm{P}$, and $\mathrm{B}$ frames would be 7,3 , and 1 . Then, the proposed method would transmit the frame sequence IPPPP in the base layer, the sequence $\mathrm{BBBBBB}$ in enhancement layer $\mathrm{I}$, and the sequence $\mathrm{BBBB}$ in enhancement layer II. Therefore, the data rate for the base layer is $167.724 \mathrm{~kb}$, that of enhancement layer $\mathrm{I}$ is $52.966 \mathrm{~kb}$, and that of enhancement layer II is $35.31 \mathrm{~kb}$.

2.2. HPWRR Scheduling. Based on the QoS requirement, the DBAM allocates the bandwidth for each user profile for different traffic types, including UGS, RT-VR, ERT-VR, NRTVR, BE, and multicast. All traffic types will be scheduled by the HPWRR scheduling algorithm shown in Figure 3. First, the BS categorizes the incoming traffic-each traffic type has its own service queue with a different scheduling algorithm. The UGS, such as voice data with a constant bit rate, has the highest priority; the HPWRR algorithm bypasses UGS traffic to the second level scheduling. In the first level scheduling, five traffic types are scheduled by different scheduling algorithms. The RT-VR traffic uses the leaky bucket [19] scheduling algorithm to control the traffic with shaping and rate limits; the ERT-VR traffic uses the earliest deadline first (EDF) [20] scheduler, depending on the packet deadline - the packets are transmitted on the basis of the smallest deadline first; the NRT-VR traffic uses the weighted fair queuing (WFQ) [21] algorithm to control the transmission rate with fairness for no packet deadline; the $\mathrm{BE}$ traffic uses the first-in first-out (FIFO) algorithm [22]; multicast traffic uses the leaky bucket algorithm to control transmission rate and shape the SVC streaming for different multicast groups with different layers. In the second level scheduling, the priority queue algorithm is used to schedule the UGS traffic and traffic from the output of the weighted round robin (WRR) algorithm, injected from the first level scheduling.

\section{Simulation Model and Performance Evaluation}

In this section, the performance of the proposed methods will be evaluated and compared in terms of throughput, delay time, and bandwidth utilization, using a network simulator 2 (NS-2) [23] based on a two-ring structure that contains seven WiMAX cells, as shown in Figure 1. Each WiMAX cell contains a WiMAX BS, which is responsible for the WiMAX new calls, moving calls, and handoff calls. The mobile users are distributed randomly in each cell with different burst profiles and move randomly with a random waypoint model [24]. In the simulation, the calls are generated by Poisson distribution and the call hold durations are exponentially distributed. The system parameters and traffic types are shown in Tables 3 and 4, respectively [2]. 
TABle 3: Mobile WiMAX PHY data rates [2].

\begin{tabular}{|c|c|c|c|}
\hline \multicolumn{4}{|c|}{ Parameters } \\
\hline \multicolumn{2}{|c|}{ System bandwidth } & \multicolumn{2}{|c|}{$10 \mathrm{MHz}$} \\
\hline \multicolumn{2}{|c|}{ BS transmission power } & \multicolumn{2}{|c|}{$0.125 \mathrm{~W}(21.0 \mathrm{dBm})$} \\
\hline \multicolumn{2}{|c|}{ Frequency } & \multicolumn{2}{|c|}{$3.5 \mathrm{GHz}$} \\
\hline \multicolumn{2}{|c|}{ FFT size } & \multicolumn{2}{|c|}{1024} \\
\hline \multicolumn{2}{|c|}{ Frame duration } & \multicolumn{2}{|c|}{5 milliseconds } \\
\hline \multicolumn{2}{|c|}{ Simulation time } & \multicolumn{2}{|c|}{3600 seconds } \\
\hline \multicolumn{2}{|c|}{ Transmission range } & \multicolumn{2}{|c|}{$1413.5 \mathrm{~m}$} \\
\hline \multicolumn{2}{|c|}{ WiMAX cells } & \multicolumn{2}{|c|}{7} \\
\hline \multicolumn{2}{|c|}{ Mobility velocity } & \multicolumn{2}{|c|}{$0 \sim 17 \mathrm{~m} / \mathrm{s}(0 \sim 60 \mathrm{~km} / \mathrm{h})$} \\
\hline Modulation & Code rate & $\begin{array}{c}\text { Downlink rate } \\
\text { (Mbps) }\end{array}$ & $\begin{array}{l}\text { Uplink rate } \\
\text { (Mbps) }\end{array}$ \\
\hline \multirow{2}{*}{ QPSK } & $1 / 2$ & 6.34 & 4.70 \\
\hline & $3 / 4$ & 9.50 & 7.06 \\
\hline \multirow{2}{*}{ 16 QAM } & $1 / 2$ & 12.67 & 9.41 \\
\hline & $3 / 4$ & 19.01 & 14.11 \\
\hline \multirow{2}{*}{64 QAM } & $2 / 3$ & 25.34 & 18.82 \\
\hline & $3 / 4$ & 28.51 & 21.17 \\
\hline
\end{tabular}

TABle 4: Parameters of traffic type [2].

\begin{tabular}{lccc}
\hline Traffic type & Data rate & Mean duration & Arrival rate \\
\hline UGS & $64 \mathrm{kbps}$ & $210 \mathrm{~s}$ & $0.1 \sim 10 \mathrm{call} / \mathrm{s}$ \\
RT-VR & $64 \sim 384 \mathrm{kbps}$ & $360 \mathrm{~s}$ & $0.1 \sim 10 \mathrm{call} / \mathrm{s}$ \\
ERT-VR & $32 \mathrm{kbps}$ & $180 \mathrm{~s}$ & $0.1 \sim 10 \mathrm{call} / \mathrm{s}$ \\
NRT-VR & $16 \mathrm{kbps}$ & $180 \mathrm{~s}$ & $0.1 \sim 10 \mathrm{call} / \mathrm{s}$ \\
BE & $10 \mathrm{kbps}$ & $30 \mathrm{~s}$ & $0.1 \sim 10 \mathrm{call} / \mathrm{s}$ \\
Multicast & $167 \sim 256 \mathrm{kbps}$ & $360 \mathrm{~s}$ & $0.1 \sim 10 \mathrm{call} / \mathrm{s}$ \\
\hline
\end{tabular}

Table 5 shows the traffic throughput of UGS, RT-VR, and ERT-VR for the default scheme, DABS1, and DABS2, respectively. DABS1 and DABS2 provide 8.26 and $9.47 \%$ improvements for UGS, respectively, while the improvements for RT-VR are 10.36 and $4.49 \%$. The possible reason that DABS1 provides more improvement than DABS2 is that DABS1 uses the ratio of the transmission throughput for each user profile while DABS2 uses the ratio of the amount of traffic of different SVC layers; therefore, the average throughput for most user traffic in DABS1 increases more than in DABS2. To achieve the best performance, DABS1 is selected in our simulation scenario.

Figure 4 shows the average delay time of all traffic types for the different arrival rates of the UGS, given that the arrival rates of the other traffic types are fixed at 0.6 calls/s. This shows that the average delay time of UGS is always lower than that of the others, because UGS traffic has the highest priority; therefore, it is always transmitted in the PQ first. The NRT-VR is scheduled by WFQ, followed by WRR with a fixed weight value, and the mean duration of the BE traffic is shorter than that of NRT-VR traffic, resulting in that an

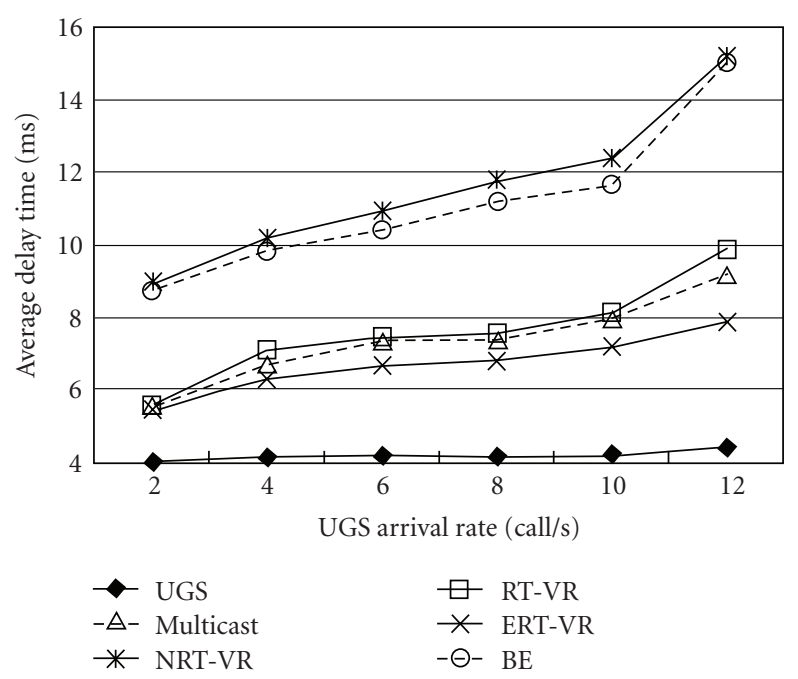

FIGURE 4: Average delay time of all traffic types for different arrival rates of the UGS, given that the arrival rates of the other traffic types are fixed at 0.6 calls/s.

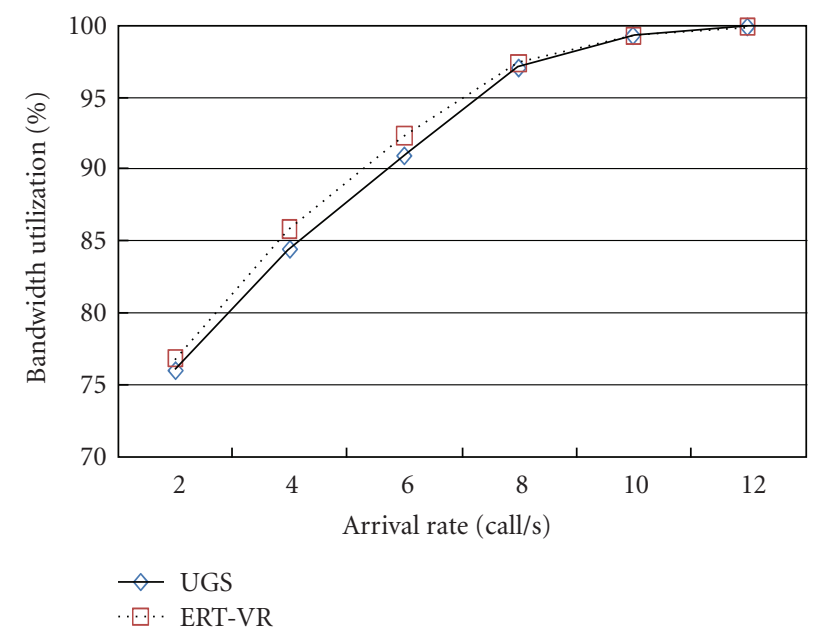

FIGURE 5: Bandwidth utilization of UGS and ERT-VR for different arrival rates, given that the arrival rates of the other traffic types are fixed at 0.6 calls/s.

average delay time for NRT-VR is higher than that of BE, even though $\mathrm{BE}$ is the lowest priority traffic.

Figure 5 shows the bandwidth utilization of UGS and ERT-VR for different arrival rates, given that the arrival rates of the other traffic types are fixed at 0.6 calls/s. The ERT-VR traffic is real-time traffic with a variable data rate, which is scheduled by the EDF; however, the UGS traffic is real-time traffic with a constant data rate, and it uses the PQ. The ERTVR bandwidth utilization is slightly higher than that of UGS traffic.

Figures 6(a) and 6(b) show the average delay time of RT-VR traffic with different arrival rates, without and with resource adjustments, given that the arrival rates of the other traffic types are fixed at 0.6 calls/s, respectively. As shown in Figure 6(a), the average delay time of RT-VR is higher than that of NRT-VR and BE for a higher arrival 
TABLE 5: Performance improvements of the proposed DABSs.

\begin{tabular}{|c|c|c|c|c|c|}
\hline \multirow{2}{*}{ Traffic } & \multirow{2}{*}{$\begin{array}{c}\text { Default MS } \\
\text { Average throughput } \\
(\mathrm{Mbps})\end{array}$} & \multicolumn{2}{|c|}{ DABS1 } & \multicolumn{2}{|c|}{ DABS2 } \\
\hline & & $\begin{array}{l}\text { Average throughput } \\
\text { (Mbps) }\end{array}$ & Improvement (\%) & $\begin{array}{l}\text { Average throughput } \\
\text { (Mbps) }\end{array}$ & Improvement (\%) \\
\hline UGS & 25.466 & 27.570 & 8.262 & 27.878 & 9.471 \\
\hline RT-VR & 26.921 & 29.711 & 10.363 & 28.129 & 4.487 \\
\hline ERT-VR & 25.833 & 28.274 & 9.491 & 28.285 & 9.492 \\
\hline
\end{tabular}

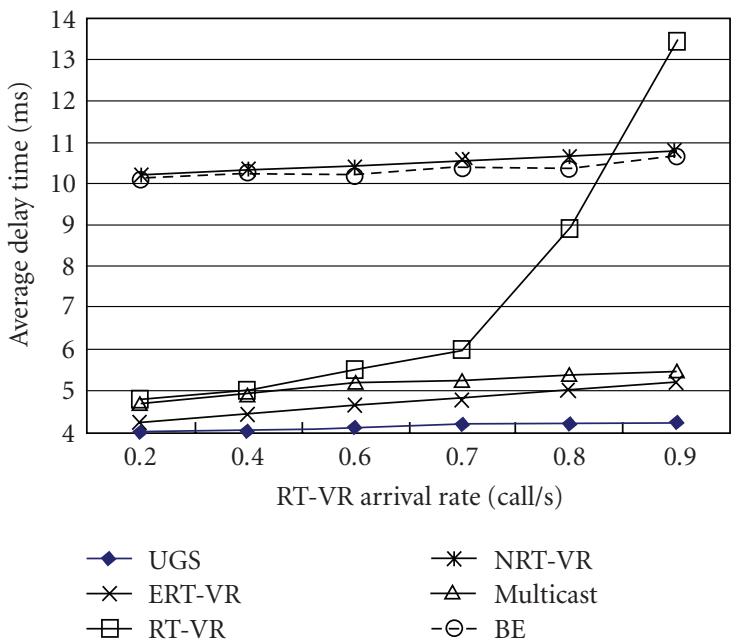

(a)

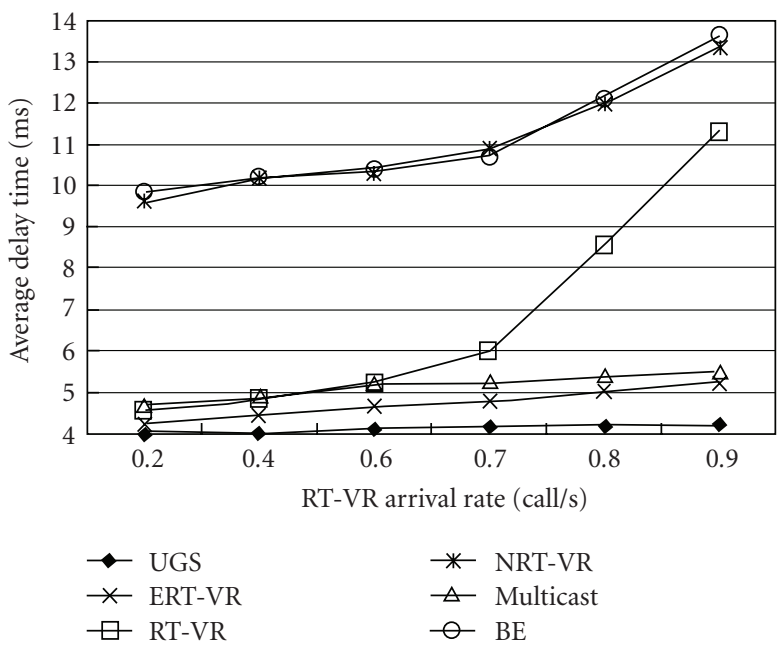

(b)

FIGURE 6: Average delay time for different arrival rates of RT-VR (a) without resource adjustment and (b) with resource adjustment, given that the arrival rates of the other traffic types are fixed at 0.6 calls/s.

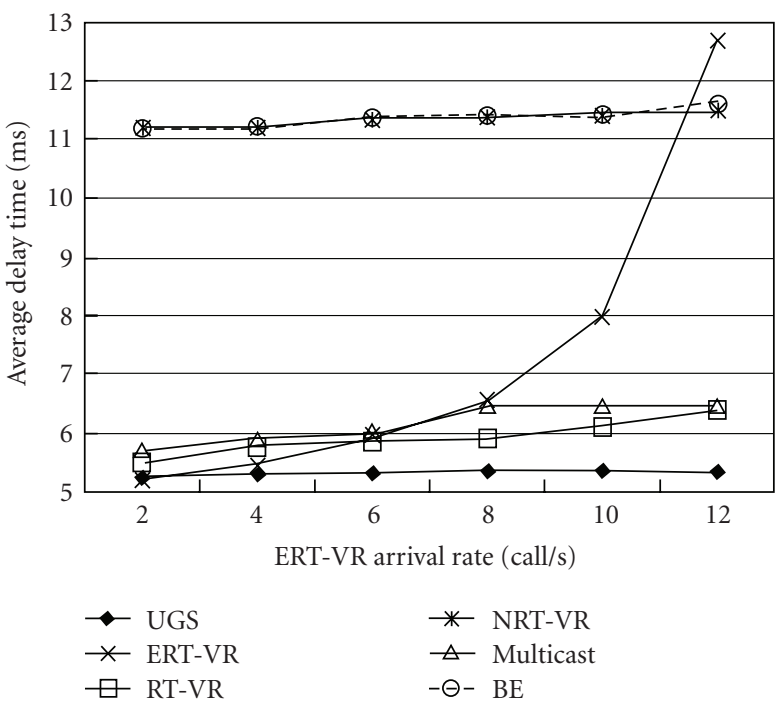

(a)

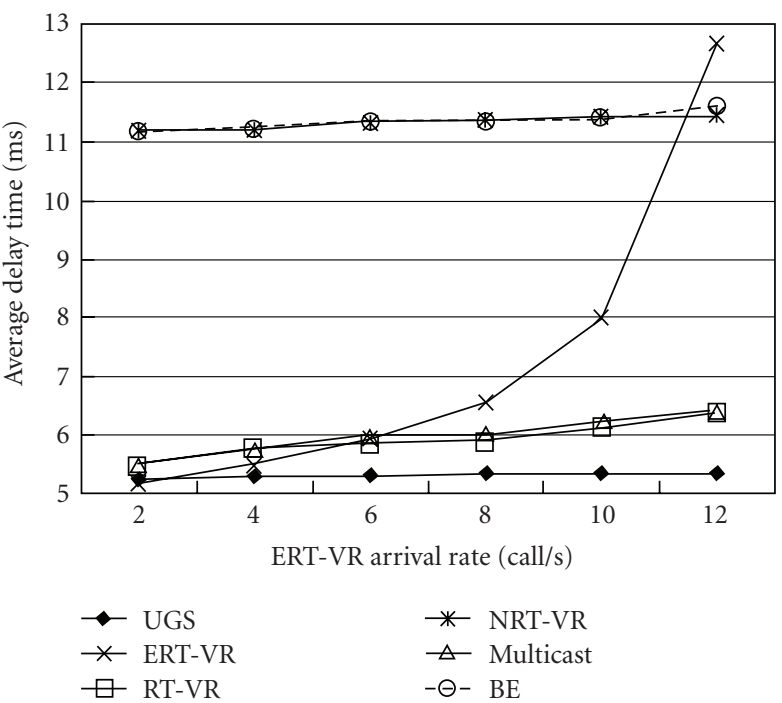

(b)

FIGURE 7: Average delay time for different arrival rates of ERT-VR (a) without resource adjustment and (b) with resource adjustment, given that the arrival rates of the other traffic types are fixed at 0.6 calls/s. 
TABLE 6

\begin{tabular}{lccc}
\hline Burst profiles & $\begin{array}{c}\text { Default } \\
\text { Demand } \\
\text { (symbols/sec) }\end{array}$ & $\begin{array}{c}\text { Proposed method } \\
\text { Demand } \\
\text { (symbols/sec) }\end{array}$ & $\begin{array}{c}\text { Improvement } \\
(\%)\end{array}$ \\
\hline $\begin{array}{l}\text { QPSK + } \\
\text { 16-QAM + } \\
\text { 64-QAM }\end{array}$ & 1334 & 1058 & 21.439 \\
QPSK + & 1334 & 1012 & 24.139 \\
$\begin{array}{l}\text { 16-QAM } \\
\text { QPSK + }\end{array}$ & 1334 & 989 & 25.862 \\
$\begin{array}{l}\text { 64-QAM } \\
\text { QPSK }\end{array}$ & 1334 & 874 & 34.482 \\
$\begin{array}{l}\text { 16-QAM + } \\
\text { 64-QAM }\end{array}$ & 667 & 621 & 7.4 \\
\hline
\end{tabular}

rates of RT-VR (i.e., above 8.3 calls/s). The reason is that the increasing arrival rate of the RT-VR results in a lack of bandwidth allocation, due to the fixed weight of the second level scheduling; this particular situation can be improved (i.e., $19.6 \%$ for 0.9 call $/ \mathrm{s}$ case) by the proposed method, as shown in Figure 6(b). When the resources are adjusted, because more resources are allotted to RT-VR traffic, the average delay for NRT-VR and BE increases and the first level scheduling of the HPWRR gives a higher weight to real-time traffic; hence, there are comparatively fewer opportunities for transmission of non-real-time traffic than for real-time traffic. Note that the DABS1 that adjusts the resources in advance has a higher delay for non-real-time and BE traffic.

Similar to the experiment for varying RT-VR arrival rates, unless ERT-VR has shorter packet size and periodic delivery, then the results are shown in Figures 7(a) and 7(b), without and with the adoption of DABS1, respectively. The ERT-VR received the second highest priority; therefore, the average delay time tends to be a normal phenomenon under a light traffic load. As the arrival rate increases, ERT-VR cannot gain sufficient bandwidth, which, in turn, results in average delay times higher than those for other traffic types. In particular, when the ETR-VR arrival rate exceeds nine calls, its delay time will be higher than that of NRT-VR and BE. Comparing Figures 7(b) and 7(a), the improvement of ERTVR using DABS1 is not obvious, due to its periodic delivery with small packet sizes and low data rates. However, the delay time of multicast with the resources adjusted improves by an average of $2.97 \%$ over that of multicast without resource adjustment-more bandwidth is released from ERT-VR.

Based on the multicast session, if the BS uses a default multicast method, it must use the most robust burst profile (e.g., QPSK) to transmit the multicast data. For our proposed method, users are classified into different multicast groups based on their received SNR to receive appropriate video layers. Table 6 shows the different combination of burst profiles for comparing the required symbols for the default and our proposed method and shows the improvements for different cases. The required number of symbols for the default method and each layer of SVC, based on different combinations of burst profiles, is shown in Figure 8.
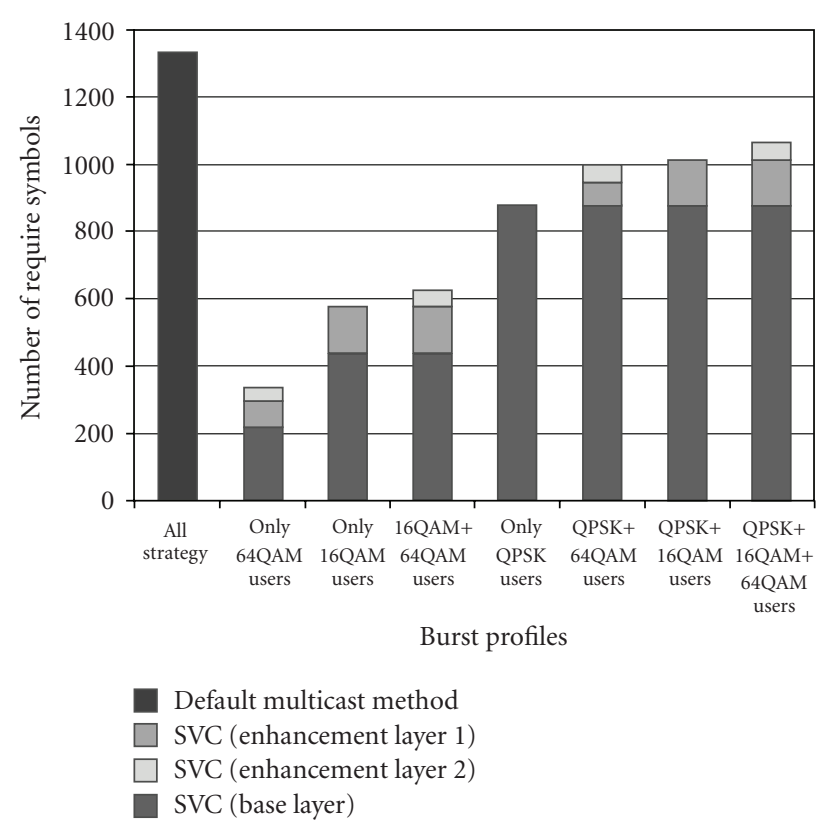

Figure 8: Comparison of different burst profiles.

\section{Conclusion}

This paper proposes a novel adaptive downlink bandwidth allocation method (DBAM) with a HPWRR scheduling algorithm for six different traffic profiles. Two adaptive resource adjustment schemes with scalable video coding (SVC) technology are used and compared in the DBAM. Simulation results show that embedding SVC technology in the DBAM improves the bandwidth utilization (i.e., $8 \%-$ $10 \%)$ and the average delay time in a mobile WiMAX network, especially for multicast traffic. Additionally, the improvements in average delay time for variable RT-VR and ERT-VR arrival rates are $19.6 \%$ for RT-VR and $2.97 \%$ for multicast. The contribution of this paper is the integration of the six traffic types, particularly the integration of multicast traffic, and efficient scheduling of the traffic types. The simulation results show that the proposed schemes effectively reduce the average delay time of the specified traffic types and improve the quality of service and total throughput. Moreover, HPWRR scheduling can precisely guarantee the QoS requirements for mobile users in a link adaptive mobile WiMAX system. Future work will be to survey the issues in an uplink scheduling algorithm.

\section{References}

[1] IEEE 802.16e-2005 and IEEE 802.16-2004/Cor 1-2005 (Amendment and Corrigendum to IEEE Std 802.16-2004), "IEEE Standard for Local and Metropolitan Area Networks Part 16: Air Interface for Fixed and Mobile Broadband Wireless Access Systems Amendment 2: Physical and Medium Access Control Layers for Combined Fixed and Mobile Operation in Licensed Bands and Corrigendum 1," 2006.

[2] "Mobile WiMAX_-Part 1: A Technical Overview and Performance Evaluation,” WiMAX Forum, August 2006. 
[3] T. Jiang, W. Xiang, H.-H. Chen, and Q. Ni, "Multicast broadcast services support in OFDMA-based WiMAX systems," IEEE Communications Magazine, vol. 45, no. 8, pp. 78-86, 2007.

[4] O. Alanen, "Multicast polling and efficient VoIP connections in IEEE 802.16 networks," in Proceedings of the 10th ACM Symposium on Modeling, Analysis, and Simulation of Wireless and Mobile Systems (MSWiM '07), pp. 289-295, October 2007.

[5] A. Lyakhov, V. Vishnevsky, and M. Yakimov, "Multicast QoS support in IEEE 802.16," in Proceedings of the 3rd ACM Workshop on Q2S and Security for Wireless and Mobile Networks (Q2SWinet'07), pp. 63-70, October 2007.

[6] J. She, F. Hou, P.-H. Ho, and L.-L. Xie, "IPTV over WiMAX: key success factors, challenges, and solutions," IEEE Communications Magazine, vol. 45, no. 8, pp. 87-93, 2007.

[7] W.-H. Kuo, T. Liu, and W. Liao, "Utility-based resource allocation for layer-encoded IPTV multicast in IEEE 802.16 (WiMAX) wireless networks," in Proceedings of the IEEE International Conference on Communications (ICC '07), pp. 1754-1759, June 2007.

[8] H.-H. Juan, H.-C. Huang, C. Huang, and T. Chiang, "Scalable video streaming over mobile WiMAX," in Proceedings of the IEEE International Symposium on Circuits and Systems (ISCAS '07), pp. 3463-3466, May 2007.

[9] ISO/IEC JTC 1/SC 29/WG 11, "Working Draft 4 of ISO/IEC. 14496-10:2005/AMD3 Scalable Video Coding, N7555,” Nice, October 2005.

[10] T. Schierl, T. Stockhammer, and T. Wiegand, "Mobile video transmission using scalable video coding," IEEE Transactions on Circuits and Systems for Video Technology, vol. 17, no. 9, pp. 1204-1217, 2007.

[11] T. Schierl, C. Hellge, S. Mirta, K. Grüneberg, and T. Wiegand, "Using H.264/AVC-based scalable video coding (SVC) for real time streaming in wireless IP networks," in Proceedings of the IEEE International Symposium on Circuits and Systems (ISCAS '07), pp. 3455-3458, May 2007.

[12] W.-F. Poon, K.-T. Lo, and J. Feng, "Performance study for streaming layered encoded videos in broadcast environment," in Proceedings of the 3rd International Conference on Information Technology and Applications (ICITA '05), pp. 722-727, July 2005.

[13] T.-K. Cheng, F.-M. Yang, J.-L. C. Wu, and I.-C. Lin, “Adaptive modulation and SVC-encoded video IPTV multicast over mobile WiMAX," in Proceedings of the IEEE-RIVF International Conference on Computing and Communication Technologies (RIVF '09), July 2009.

[14] C. Y. Huang, H.-H. Juan, M.-S. Lin, and C.-J. Chang, "Radio resource management of heterogeneous services in mobile WiMAX systems," IEEE Wireless Communications, vol. 14, no. 1, pp. 20-26, 2007.

[15] Y. Zhang and P. G. Harrison, "Performance of a priorityweighted round robin mechanism for differentiated service networks," in Proceedings of the 16th International Conference on Computer Communications and Networks (ICCCN '07), pp. 1198-1203, August 2007.

[16] W. Shen and Q.-A. Zeng, "A novel decision strategy of vertical handoff in overlay wireless networks," in Proceedings of the 5th IEEE International Symposium on Network Computing and Applications (NCA '06), pp. 227-230, July 2006.

[17] I-S. Hwang, B. J. Hwang, and C. W. Huang, "Framebased adaptive uplink scheduling algorithm in OFDMA-based WiMAX networks," in Proceedings of the 10th International Symposium on Pervasive Systems, Algorithms and Networks, pp. 278-283, December 2009.
[18] G. J. Sullivan, P. Topiwala, and A. Luthra, "The H.264/AVC advanced video coding standard: overview and introduction to the fidelity range extensions," in Applications of Digital Image Processing XXVII, vol. 5558 of Proceedings of SPIE, pp. 454-474, Denver, Colo, USA, August 2004.

[19] G. Rizzo and J.-Y. L. Boudec, "Generalization of the RIN result to heterogeneous networks of aggregate schedulers and leaky bucket constrained flows," in Proceedings of the 15th IEEE International Conference on Networks (ICON '07), pp. 388393, November 2007.

[20] F. Zhang and A. Burns, "Analysis of hierarchical EDF preemptive scheduling," in Proceedings of the 28th IEEE International Real-Time Systems Symposium (RTSS '07), pp. 423-434, December 2007.

[21] J. C. R. Bennett and H. Zhang, "WF²Q: worst-case fair weighted fair queueing," in Proceedings of the 15th Annual Joint Conference of the IEEE Computer and Communications Societies (INFOCOM '96), pp. 120-128, March 1996.

[22] A. Iera, A. Molinaro, and S. Pizzi, "Channel-aware scheduling for QoS and fairness provisioning in IEEE 802.16/WiMAX broadband wireless access systems," IEEE Network, vol. 21, no. 5, pp. 34-41, 2007.

[23] "Network Simulator 2," http://www.isi.edu/nsnam/ns/.

[24] “The Random Trip Mobility Model," http://icawww1.epfl.ch/ RandomTrip/. 

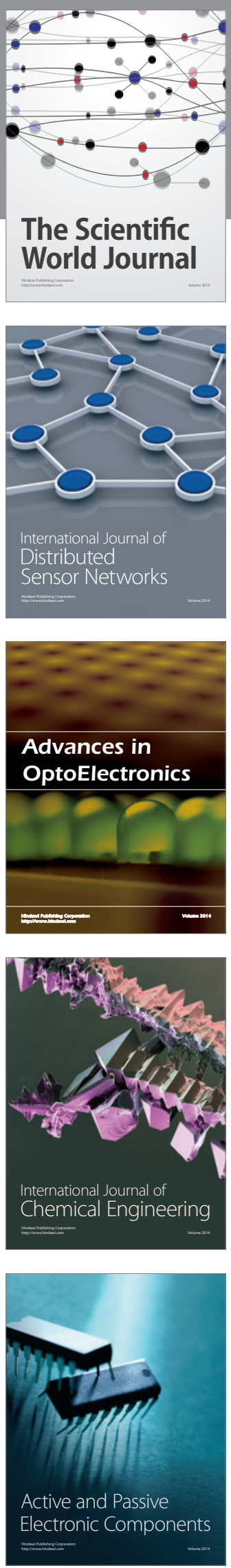
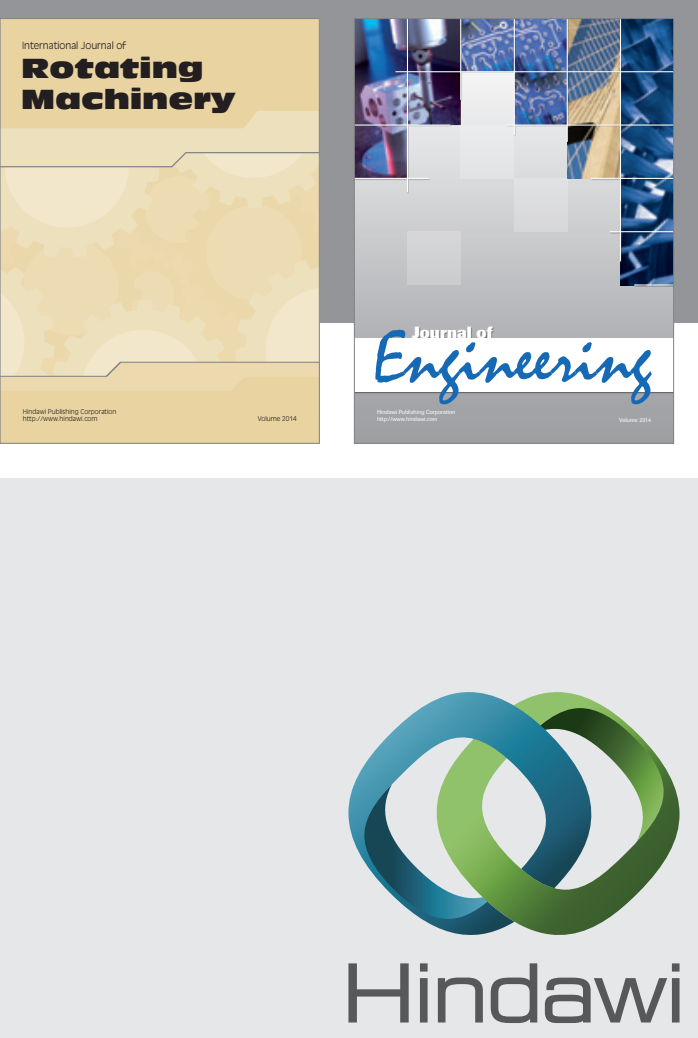

Submit your manuscripts at

http://www.hindawi.com
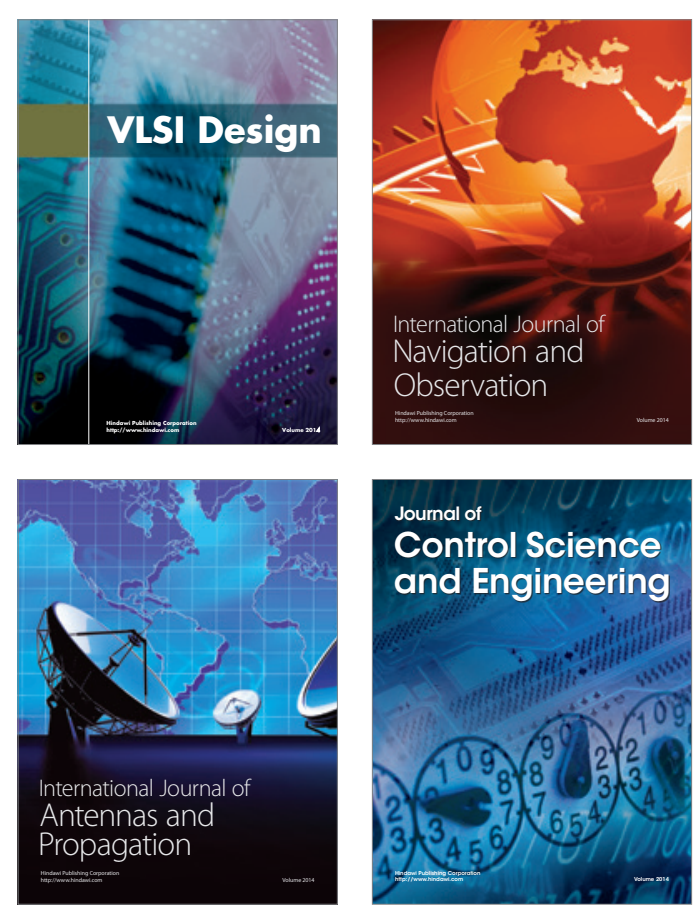
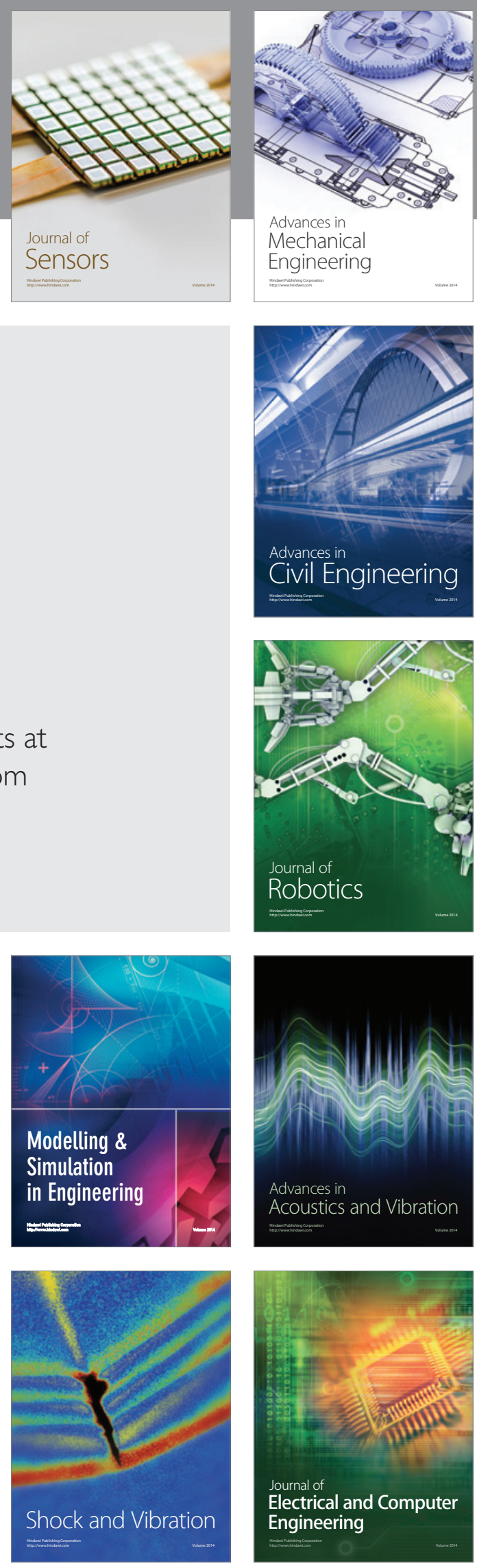